\title{
Основные квазиматричные логики
}

\author{
(C) Ю.В. Ивлев
}

МГУ им. М.В. Ломоносова, Москва, 119991, Россия

Известны возможности применения матричных логик вне области логики. Это релейно-контактные схемы и абстрактные автоматы. В случае применения матричных логик к описанию автоматов зависимости между сигналами на входе автомата, его состоянием и действием на выходе выражаются посредством функций. Использование квазиматричной логики позволит выражать эту зависимость посредством квазифункций. Поскольку частным случаем квазифункции является функция, появляется возможность создания новых видов автоматических устройств, решающих более широкий спектр задач. Однако для квазиматричных логик актуальна проблема разрешимости, что не позволяет применять квазиматричную логику в указанной сфере. В статье предложено решение этой проблемы.

Ключевые слова: матричная логика, квазиматричная логика, квазифункция, семантическая полнота, разрешимость исчисления

Матричная логика. При семантическом построении этой логики используется понятие матрицы. Матрица - это множество $\left(\mathbf{W}, \mathbf{V}, \mathbf{f}_{\mathbf{1}}\right.$, $\left.\mathbf{f}_{\mathbf{2}}, \ldots\right)$, где $\mathbf{W}$ - непустое множество элементов матрицы, $\mathbf{V}$ - множество выделенных элементов $(\mathbf{V} \subset \mathbf{W}), \mathbf{f}_{\mathbf{1}}, \mathbf{f}_{2}, \ldots-$ функции, являющиеся интерпретациями логических терминов. Например, для классической логики высказываний матрица - это множество $\left(\mathbf{W}, \mathbf{V}, \mathbf{f}^{\mathbf{1}}\right.$, $\left.\mathbf{f}^{\mathbf{2}}\right)$, где $\mathbf{W}-\{\mathbf{u}, \boldsymbol{\pi}\} ; \mathbf{V}-\{\mathbf{u}\} ; \mathbf{f}^{\mathbf{1}}-$ одноместная функция, соответствующая отрицанию; $\mathbf{f}^{2}$ - двухместная функция, соответствующая, например, конъюнкции (отрицание и конъюнкция - полная система связок). Здесь «и» - значение «истина», а «л» — «ложь».

Квазиматричная логика. Основным понятием этой логики, предложенной автором данной статьи в 1970-х гг. ХХ в. [1], является понятие квазиматрицы [2-5].

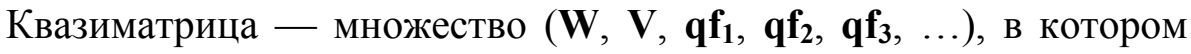
$\mathbf{q} \mathbf{f}_{1}, \mathbf{q} \mathbf{f}_{2}, \mathbf{q \mathbf { f } _ { 3 }}, \ldots$ - квазифункции. Квазифункция — это соответствие, в силу которого какой-то элемент подмножества множества, являющегося областью определения функции, соотносится с каким-то элементом подмножества множества, представляющего собой областью значений функции.

Примеры. 1. Функиия. Пусть областью ее определения является множество $\{\mathbf{a}, \mathbf{b}, \mathbf{c}\}$, а областью значений - множество $\{\mathbf{d}, \mathbf{s}, \mathbf{h}\}$. Функция может быть задана разными парами элементов этих множеств, например $\{(\mathbf{a}, \mathbf{d}),(\mathbf{b}, \mathbf{s}),(\mathbf{c}, \mathbf{h})\}$. 
2. Квазифункиия. Пусть область определения и область значений квазифункции одни и те же. Квазифункция может быть представлена множеством $\{($ либо $(\mathbf{a}, \mathbf{d})$, либо $(\mathbf{a}, \mathbf{s})$, либо $(\mathbf{b}, \mathbf{d})$, либо $(\mathbf{b}, \mathbf{s})),(\mathbf{c}, \mathbf{h})\}$.

Квазиматричная логика $\mathbf{S}_{\min }$. К языку классической логики высказываний, содержащему логические термины $\neg$ (отрицание) и $\supset$ ((материальная) импликация), скобки и пропозициональные переменные $\mathbf{p}, \mathbf{q}, \mathbf{r}, \mathbf{s}, \mathbf{p}_{1}, \mathbf{q}_{1}, \ldots$, добавляются модальные термины (необходимость) и $\diamond$ (возможность). Определение формулы обычное.

Квазиматрица логики $\mathbf{S}_{\mathbf{m i n}}$ :

$$
\left(\{\mathbf{n}, \boldsymbol{л}\},\{\mathbf{n}\}, \mathbf{f}^{\mathbf{1}}, \mathbf{f}^{\mathbf{2}}, \mathbf{q} \mathbf{f}_{\mathbf{1}}, \mathbf{q} \mathbf{f}_{\mathbf{2}}\right),
$$

где $\mathbf{f}^{\mathbf{1}}$ - одноместная функция (частный случай квазифункции), посредством которой определяется отрицание; $\mathbf{f}^{2}$ - двухместная функция (так же частный случай квазифункции), посредством которой

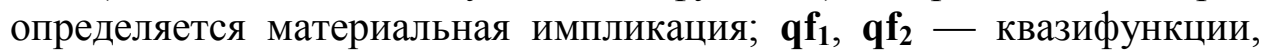
посредством которых определяются знаки необходимости и возможности. Знаки отрицания и импликации определяются обычным образом.

Определения знаков необходимости и возможности:

$$
\begin{aligned}
|\mathbf{A}|=\boldsymbol{л} \Leftrightarrow|\square \mathbf{A}|=\boldsymbol{л} ;|\mathbf{A}|=\mathbf{n} \Rightarrow|\square \mathbf{A}| \in\{\mathbf{n}, \boldsymbol{л}\} ; \\
|\mathbf{A}|=\mathbf{n} \Rightarrow|\nabla \mathbf{A}|=\mathbf{n} ;|\mathbf{A}|=\boldsymbol{л} \Rightarrow|\nabla \mathbf{A}| \in\{\mathbf{n}, \boldsymbol{л}\} .
\end{aligned}
$$

Аналогичные определения модальных терминов даны Решером [6], но он не построил квазиматричную логику, поскольку в его логике $(\mathbf{Q})$ выделенными значениями являются «истина» $(\mathbf{t})$ и «то ли истина, то ли ложь» $(\mathbf{t}, \mathbf{f})$.

Выражение $|\mathbf{G}|$ читается: «значение формулы $\mathbf{G} » ; \mid$ | - сокращение для последовательности квазифункций $\left(\boldsymbol{\varphi}, \mathbf{q \mathbf { f } _ { 1 }}, \mathbf{q \mathbf { f } _ { 2 }}, \mathbf{q \mathbf { f } _ { 3 }}, \ldots\right) ; \boldsymbol{\varphi}-$ функция (частный случай квазифункции) приписывания значений пропозициональным переменным (функция интерпретации переменных). Каждая из (квази)функций последовательности $\left(\mathbf{q} \mathbf{f}_{\mathbf{1}}, \mathbf{q \mathbf { f } _ { 2 }}, \mathbf{q \mathbf { f } _ { 3 }}, \ldots\right)$ применяется к соответствующему логическому термину. Если в формуле не встречается логический термин, который интерпретируется (квази)функцией из этой последовательности, то она не применяется.

Исчисление $\mathbf{S}_{\mathbf{m i n}}$. K исчислению классической логики высказываний добавляются две схемы аксиом: $\square \mathrm{A} \supset \mathrm{A}, \mathrm{A} \supset \diamond \mathrm{A}$. Определения доказательства, теоремы и вывода не изменяются [7].

Основные четырехзначные квазиматричные логики. Из построенных автором данной статьи четырехзначных модальных логик [8, с. 74-128] выделим в качестве основных квазиматричных логические системы $\mathbf{S}_{\mathbf{a}}^{+}, \mathbf{S}_{\mathbf{3}}^{+}, \mathbf{S}_{\mathbf{n}}^{+}, \mathbf{S}_{\mathbf{K}}^{+}, \mathbf{S}_{\text {л }}^{+}$. В них все логические термины, кроме отрицания, интерпретируются посредством квазифункций. Отрицание интерпретируется с помощью функции, являющейся частным случаем квазифункции. Во всех этих логиках $\mathbf{W}$ есть множество 
значений $\left\{\mathbf{t}^{\mathbf{n}}, \mathbf{t}^{\mathbf{c}}, \mathbf{f}^{\mathbf{i}}, \mathbf{f}^{\mathbf{c}}\right\}$. Множество выделенных значений $\mathbf{V}$ есть $\left\{\mathbf{t}^{\mathbf{n}}\right.$, $\left.\mathbf{t}^{\mathbf{c}}\right\} ; \mathbf{t}^{\mathbf{n}}$ читается как «необходимая истина». Высказывание имеет это значение, если описываемое им положение дел имеет место в действительности, как и это положение однозначно детерминировано.

Примеры. 1. Определенные генные аномалии обязательно приводят к определенному заболеванию (заболевание имеет место, и оно однозначно детерминировано).

2. Прекращение подачи электричества обязательно приводит к остановке работы станка (станок не работает, и это однозначно детерминировано отсутствием подачи электричества).

$\mathbf{t}^{\mathbf{c}}$ читается как «случайная истина». Высказывание имеет это значение, если описываемое им положение дел имеет место в действительности и не детерминировано однозначно.

Примеры. 1. Определенные генные аномалии иногда приводят к определенному заболеванию, а иногда не приводят, но в данном случае это заболевание имеет место (но оно однозначно не детерминировано генными аномалиями).

2. Снижение напряжения электрического тока иногда приводит к остановке работы механизма, а иногда не приводит (механизм не работает, и это не детерминировано однозначно снижением напряжения электрического тока).

$\mathbf{f}^{i}$ - «необходимая ложь» (ложно и невозможно), $\mathbf{f}^{\mathfrak{c}}$ - «случайная ложь» (положение дел не имеет места, и его отсутствие не детерминировано однозначно).

Квазиматричная логика $\mathbf{S}_{\mathbf{K}}^{+}$. Язык тот же, что и в логике $\mathbf{S}_{\mathbf{m i n}}$. Квазиматрица - $\left(\left\{\mathbf{t}^{\mathbf{n}}, \mathbf{t}^{\mathbf{c}}, \mathbf{f}^{\mathbf{i}}, \mathbf{f}^{\mathbf{c}}\right\},\left\{\mathbf{t}^{\mathbf{n}}, \mathbf{t}^{\mathbf{c}}\right\}, \mathbf{f}^{\mathbf{1}}, \mathbf{q} \mathbf{f}^{\mathbf{2}}, \mathbf{q} \mathbf{f}_{1}, \mathbf{q} \mathbf{f}_{2}\right)$. Посредством функции $\mathbf{f}^{1}$ отрицание определяется следующим образом:

$$
\begin{aligned}
& |\neg \mathbf{A}|=\mathbf{t}^{\mathbf{n}} \Leftrightarrow|\mathbf{A}|=\mathbf{f}^{\mathbf{i}} ;|\neg \mathbf{A}|=\mathbf{t}^{\mathbf{c}} \Leftrightarrow|\mathbf{A}|=\mathbf{f}^{\mathbf{c}} ; \\
& |\neg \mathbf{A}|=\mathbf{f}^{\mathbf{i}} \Leftrightarrow|\mathbf{A}|=\mathbf{t}^{\mathbf{n}} ;|\neg \mathbf{A}|=\mathbf{f}^{\mathbf{c}} \Leftrightarrow|\mathbf{A}|=\mathbf{t}^{\mathbf{c}} .
\end{aligned}
$$

Квазифункция $\mathbf{q f}^{\mathbf{2}}$ является интерпретацией импликации:

$|\mathbf{A} \supset \mathbf{B}|=\mathbf{f}^{\mathbf{c}} \Leftrightarrow\left(|\mathbf{A}|=\mathbf{t}^{\mathbf{n}}\right.$ и $\left.|\mathbf{B}|=\mathbf{f}^{\mathbf{c}}\right)$ или $\left(|\mathbf{A}|=\mathbf{t}^{\mathbf{c}}\right.$ и $\left.|\mathbf{B}|=\mathbf{f}^{\mathbf{i}}\right)$;

$|\mathbf{A} \supset \mathbf{B}|=\mathbf{f}^{\mathbf{i}} \Leftrightarrow|\mathbf{A}|=\mathbf{t}^{\mathbf{n}}$ и $|\mathbf{B}|=\mathbf{f}^{\mathbf{i}}$; если или $\left(|\mathbf{A}|=\mathbf{t}^{\mathbf{n}}\right.$ и $\left.|\mathbf{B}|=\mathbf{t}^{\mathbf{c}}\right)$,

или $\left(|\mathbf{A}|=\mathbf{f}^{\mathbf{c}}\right.$ и $\left.|\mathbf{B}|=\mathbf{f}^{\mathbf{i}}\right)$, то $|\mathbf{A} \supset \mathbf{B}|=\mathbf{t}^{\mathbf{c}}$;

если $|\mathbf{A}|=\mathbf{f}^{\mathbf{i}}$ или $|\mathbf{B}|=\mathbf{t}^{\mathbf{n}}$, то $|\mathbf{A} \supset \mathbf{B}|=\mathbf{t}^{\mathbf{n}}$;

если или $|\mathbf{A}|=|\mathbf{B}|=\mathbf{t}^{\mathbf{c}}$, или $\left(|\mathbf{A}|=\mathbf{f}^{\mathfrak{c}}\right.$ и $\left.|\mathbf{B}|=\mathbf{t}^{\mathbf{c}}\right)$,

или $\left.|\mathbf{A}|=|\mathbf{B}|=\mathbf{f}^{\mathbf{c}}\right)$, то $|\mathbf{A} \supset \mathbf{B}| \in\left\{\mathbf{t}^{\mathbf{n}}, \mathbf{t}^{\mathbf{c}}\right\}$.

Определение квазифункции qf:

$$
\begin{aligned}
& |\mathbf{A}|=\mathbf{t}^{\mathbf{n}} \Rightarrow|\square \mathbf{A}|=\mathbf{t}^{\mathbf{n}} ;|\mathbf{A}|=\mathbf{t}^{\mathbf{c}} \Rightarrow|\square \mathbf{A}| \in\left\{\mathbf{f}^{\mathbf{c}}, \mathbf{f}^{\mathbf{i}}\right\} ; \\
& |\mathbf{A}|=\mathbf{f}^{\mathfrak{c}} \Rightarrow|\square \mathbf{A}| \in\left\{\mathbf{f}^{\mathfrak{c}}, \mathbf{f}^{\mathbf{i}}\right\} ;|\mathbf{A}|=\mathbf{f}^{\mathbf{i}} \Rightarrow|\square \mathbf{A}|=\mathbf{f}^{\mathbf{i}} .
\end{aligned}
$$

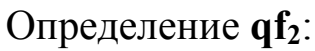


если $|\mathbf{A}|=\mathbf{t}^{\mathbf{c}}$ или $|\mathbf{A}|=\mathbf{f}^{\mathbf{c}}$,

то $|\nabla \mathbf{A}| \in\left\{\mathbf{t}^{\mathbf{n}}, \mathbf{t}^{\mathbf{c}}\right\} ;|\mathbf{A}|=\mathbf{t}^{\mathbf{n}} \Rightarrow|\nabla \mathbf{A}|=\mathbf{t}^{\mathbf{n}} ;|\mathbf{A}|=\mathbf{f}^{\mathbf{i}} \Rightarrow|\nabla \mathbf{A}|=\mathbf{f}^{\mathbf{i}}$.

Исчисление $\mathbf{S}_{\boldsymbol{\kappa}}^{+}$. K исчислению классической логики высказываний добавляются схемы аксиом

$$
\begin{aligned}
& \square \mathbf{A} \supset \mathbf{A} ; \square \mathbf{A} \supset \square \square \mathbf{A} ; \diamond \square \mathbf{A} \supset \diamond \mathbf{A} ; \square \mathbf{A} \supset \square \vee \mathbf{A} ; \diamond \diamond \mathbf{A} \supset \diamond \mathbf{A} ; \neg \square \neg \mathbf{A} \supset \diamond \mathbf{A} ; \\
& \diamond \mathbf{A} \supset \neg \square \neg \mathbf{A} ; \\
& \neg \nabla \mathbf{A} \supset \square(\mathbf{A} \supset \mathbf{B}) ; \square \mathbf{B} \supset \square(\mathbf{A} \supset \mathbf{B}) ; \forall \mathbf{B} \supset \diamond(\mathbf{A} \supset \mathbf{B}) ; \diamond \neg \mathbf{A} \supset \diamond(\mathbf{A} \supset \mathbf{B}) ; \\
& \diamond(\mathbf{A} \supset \mathbf{B}) \supset(\square \mathbf{A} \supset \diamond \mathbf{B}) ; \\
& \square(\mathbf{A} \supset \mathbf{B}) \supset(\square \mathbf{A} \supset \square \mathbf{B}) ; \square(\mathbf{A} \supset \mathbf{B}) \supset(\vee \mathbf{A} \supset \diamond \mathbf{B})
\end{aligned}
$$

и правило вывода (для облегчения доказательств) - замена двойного отрицания формулы на саму формулу, и наоборот. Определения доказательства, теоремы и вывода обычные.

Метатеорема 1. Каждая теорема является общезначимой формулой. Доказательство опускается.

Метатеорема 2. Каждая общезначимая формула является теоремой.

Сначала автор статьи обобщил метод Хенкина и доказал обобщенным методом эту метатеорему. Однако обобщенный метод Хенкина не решает проблему разрешимости исчисления, что не позволяет использовать квазиматричную логику при проектировании и использовании автоматических устройств. Здесь автор предлагает другой разработанный им метод, решающий проблему разрешимости. Он является обобщением метода Кальмара.

Замечание. Задачи разработать такой метод и применить его для проектирования автоматических устройств сформулированы автором в работе [3, с. 209].

Для доказательства метатеоремы 2 доказывается следующая лемма.

Лемма. Пусть $\mathbf{F}-$ формула, $\boldsymbol{\alpha}_{1}, \ldots, \boldsymbol{\alpha}_{\mathbf{n}}$ - все различные переменные, входящие в $\mathbf{F}$, а $\boldsymbol{\beta}_{1}, \ldots, \boldsymbol{\beta}_{\mathbf{n}}$ - значения этих переменных. Пусть $\mathbf{B}_{\mathbf{i}}$ есть $\square \boldsymbol{\alpha}_{\mathbf{i}}, \boldsymbol{\alpha}_{\mathrm{i}} \& \diamond \neg \boldsymbol{\alpha}_{\mathrm{i}}, \neg \nabla \boldsymbol{\alpha}_{\mathbf{i}}$ или $\neg \boldsymbol{\alpha}_{\mathbf{i}} \& \diamond \boldsymbol{\alpha}_{\mathbf{i}}$ в зависимости от того, что есть $\boldsymbol{\beta}_{\mathrm{i}}: \mathbf{t}^{\mathbf{n}}, \mathbf{t}^{\mathbf{c}}, \mathbf{f}^{\mathbf{i}}$ или $\mathbf{f}^{\mathbf{c}}$. Пусть $\mathbf{F}^{\prime}$ есть $\square \mathbf{F}_{\mathbf{i}}, \mathbf{F}_{\mathrm{i}} \& \diamond \neg \mathbf{F}_{\mathbf{i}}, \neg \diamond \mathbf{F}_{\mathbf{i}}$ или $\neg \mathbf{F}_{\mathrm{i}} \& \diamond \mathbf{F}_{\mathbf{i}}$, в зависимости от того, принимает ли $\mathbf{F}$ значение $\mathbf{t}^{\mathbf{n}}, \mathbf{t}^{\mathbf{c}}, \mathbf{f}^{\mathbf{i}}$ или $\mathbf{f}^{\mathbf{c}}$ при значениях $\beta_{1}, \ldots, \beta_{\mathbf{n}}$ переменных $\alpha_{1}, \ldots, \boldsymbol{\alpha}_{\mathbf{n}}$ во всех альтернативных интерпретациях, образованных на основе данной интерпретации переменных; пусть $\mathbf{F}^{\prime}$ есть $\left.\square \mathbf{F}_{\mathbf{i}} \vee\left(\mathbf{F}_{\mathbf{i}} \& \diamond \neg \mathbf{F}_{\mathbf{i}}\right), \square \mathbf{F}_{\mathbf{i}} \vee \neg \checkmark \mathbf{F}_{\mathbf{i}}, \quad\left(\mathbf{F}_{\mathbf{i}} \& \diamond \neg \mathbf{F}_{\mathbf{i}}\right)\right) \neg \neg \mathbf{F}_{\mathbf{i}}$, $\left(\square \mathbf{F}_{\mathbf{i}} \vee\left(\mathbf{F}_{\mathbf{i}} \& \diamond \neg \mathbf{F}_{\mathbf{i}}\right)\right) \vee \neg \diamond \mathbf{F}_{\mathbf{i}}$ и т. д., в зависимости от того, принимает ли формула $\mathbf{F}$ соответственно в некоторых альтернативных интерпретациях, образованных на основе данной интерпретации переменных, значение $\mathbf{t}^{\mathbf{n}}$, а в некоторых - значение $\mathbf{t}^{\mathbf{c}}$; в некоторых - $\mathbf{t}^{\mathbf{n}}$, а в неко- 
торых - $\mathbf{f}^{\mathbf{i}}$; в некоторых - $\mathbf{t}^{\mathbf{c}}$, а в некоторых - $\mathbf{f}^{\mathbf{i}} ;$ в некоторых - $\mathbf{t}^{\mathbf{n}}$, в некоторых - $\mathbf{t}^{\mathbf{c}}$, а в некоторых - $\mathbf{f}^{\mathbf{i}}$ и т. д. Тогда $\mathbf{B}_{1}, \ldots, \mathbf{B}_{\mathbf{n}} \Rightarrow \mathbf{F}^{\prime}$.

Лемма доказывается возвратной индукцией по числу вхождений логических терминов в формулу $\mathbf{F}$.

Базис. Формула $\mathbf{F}$ не содержит логических терминов. Очевидное доказательство опускается.

Индукционное допущзение. Утверждение верно для формул, имеющих не более $\mathbf{s}$ вхождений логических терминов.

Индукционный маг. Случай 1. Пусть s+1-м вхождением логических терминов в формулу $\mathbf{F}$ является вхождение знака отрицания. Формула $\mathbf{F}$ есть $\neg \mathbf{L}$.

Пусть $\mathbf{F}$ имеет значение $\mathbf{t}^{\mathbf{n}}$ в каждой альтернативной интерпретации, образованной на основе данной интерпретации переменных. Тогда $\mathbf{L}$ имеет значение $\mathbf{f}^{\mathbf{i}}$ в каждой альтернативной интерпретации, образованной на основе данной интерпретации переменных. В силу индукционного допущения $\mathbf{B}_{\mathbf{1}}, \ldots, \mathbf{B}_{\mathbf{n}} \Rightarrow \neg \diamond \mathbf{L} ; \neg \backslash \mathbf{L} \supset \square \neg \mathbf{L}-$ теорема. (Используем схему аксиом $\neg \square \neg \mathbf{A} \supset \diamond \mathbf{A}$.) Тогда $\mathbf{B}_{1}, \ldots, \mathbf{B}_{\mathbf{n}} \Rightarrow \square \neg \mathbf{L}$.

Пусть $\mathbf{F}$ имеет значение $\mathbf{f}^{\mathbf{1}}$ в каждой альтернативной интерпретации, образованной на основе данной интерпретации. Тогда $\mathbf{L}$ имеет значение $\mathbf{t}^{\mathbf{n}}$ в каждой альтернативной интерпретации. В силу индукционного допущения $\mathbf{B}_{1}, \ldots, \mathbf{B}_{\mathbf{n}} \Rightarrow \square \mathbf{L}$. Тогда $\mathbf{B}_{1}, \ldots, \mathbf{B}_{\mathbf{n}} \Rightarrow \neg \nabla \mathbf{L}$. Используем схему аксиом $\diamond \mathbf{A} \supset \neg \square \neg \mathbf{A}$ и правило замены $\neg \mathbf{A}$ на $\mathbf{A}$, и наоборот.

Пусть $\mathbf{F}$ имеет значение $\mathbf{t}^{\mathbf{c}}$ в каждой альтернативной интерпретации, образованной на основе данной интерпретации. Тогда $\mathbf{L}$ имеет значение $\mathbf{f}^{\mathbf{c}}$ в каждой альтернативной интерпретации. В силу индукционного допущения $\mathbf{B}_{1}, \ldots, \mathbf{B}_{\mathbf{n}} \Rightarrow \neg \mathbf{L} \& \diamond \mathbf{L}$.

Отсюда $\mathbf{B}_{\mathbf{1}}, \ldots, \mathbf{B}_{\mathbf{n}} \Rightarrow \neg \mathbf{L} \& \curlyvee_{\neg} \mathbf{L}$.

Пусть $\mathbf{F}$ имеет значение $\mathbf{f}^{\mathfrak{c}}$ в каждой альтернативной интерпретации, образованной на основе данной интерпретации. Тогда $\mathbf{L}$ имеет значение $\mathbf{t}^{\mathbf{c}}$ в каждой альтернативной интерпретации. В силу индукционного допущения $\mathbf{B}_{1}, \ldots, \mathbf{B}_{\mathbf{n}} \Rightarrow \mathbf{L} \& \curlyvee \mathbf{L}$.

Отсюда $\mathbf{B}_{1}, \ldots, \mathbf{B}_{\mathbf{n}} \Rightarrow \neg \neg \mathbf{L} \& \downarrow \neg \mathbf{L}$.

Пусть $\mathbf{F}$ имеет значение $\mathbf{t}^{\mathbf{n}}$ в некоторых альтернативных интерпретациях, образованных на основе данной интерпретации, а в некоторых - значение $\mathbf{t}^{\mathbf{c}}$. Тогда $\mathbf{L}$ в некоторых альтернативных интерпретациях имеет значение $\mathbf{f}^{\mathbf{i}}$, а в некоторых - $\mathbf{f}^{\mathbf{c}}$. В силу индукционного допущения $\mathbf{B}_{\mathbf{1}}, \ldots, \mathbf{B}_{\mathbf{n}} \Rightarrow \neg \diamond \mathbf{L} \vee \neg \mathbf{L} \& \diamond \mathbf{L}$.

Поскольку $\neg \backslash \mathbf{L} \Rightarrow \square \neg \mathbf{L}$ и $\neg \mathbf{L} \& \diamond \mathbf{L} \Rightarrow \neg \mathbf{L} \& \diamond \neg \neg \mathbf{L}$,

имеем $\mathbf{B}_{\mathbf{1}}, \ldots, \mathbf{B}_{\mathbf{n}} \Rightarrow \square \neg \mathbf{L} \vee(\neg \mathbf{L} \& \diamond \neg \neg \mathbf{L})$.

Пусть $\mathbf{F}$ имеет значение $\mathbf{t}^{\mathbf{n}}$ в некоторых альтернативных интерпретациях, образованных на основе данной интерпретации, а в неко- 
торых - значение $\mathbf{f}^{\mathbf{i}}$. Тогда $\mathbf{L}$ в некоторых альтернативных интерпретациях имеет значение $\mathbf{f}^{\mathbf{i}}$, а в некоторых - $\mathbf{t}^{\mathbf{n}}$. В силу индукционного допущения $\mathbf{B}_{1}, \ldots, \mathbf{B}_{\mathbf{n}} \Rightarrow \neg \nabla \mathbf{L} \vee \square \mathbf{L}$. Тогда $\mathbf{B}_{1}, \ldots, \mathbf{B}_{\mathbf{n}} \Rightarrow \square \neg \mathbf{L} \vee \neg \neg \neg \mathbf{L}$.

Пусть $\mathbf{F}$ имеет значение $\mathbf{t}^{\mathbf{c}}$ в некоторых альтернативных интерпретациях, образованных на основе данной интерпретации, а в некоторых - значение $\mathbf{f}^{\mathbf{c}}$. Тогда $\mathbf{L}$ в некоторых альтернативных интерпретациях имеет значение $\mathbf{f}^{\mathbf{c}}$, а в некоторых - $\mathbf{t}^{\mathbf{c}}$. В силу индукционного допущения $\mathbf{B}_{\mathbf{1}}, \ldots, \mathbf{B}_{\mathbf{n}} \Rightarrow(\neg \mathbf{L} \& \diamond \mathbf{L}) \vee\left(\mathbf{L} \& \downarrow_{-} \mathbf{L}\right)$.

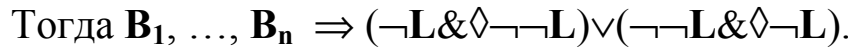

Доказательство остальных подслучаев этого случая опускается.

Случай 2. Пусть $\mathbf{s}+\mathbf{1 - м ~ в х о ж д е н и е м ~ л о г и ч е с к и х ~ т е р м и н о в ~ в ~ ф о р м у л у ~}$ $\mathbf{F}$ является вхождение знака необходимости. Формула $\mathbf{F}$ есть $\mathbf{L}$.

Пусть $\mathbf{F}$ имеет значение $\mathbf{t}^{\mathbf{n}}$ в каждой альтернативной интерпретации, образованной на основе данной интерпретации переменных. Тогда $\mathbf{L}$ имеет то же значение в каждой альтернативной интерпретации, образованной на основе данной интерпретации переменных. В силу индукционного допущения $\mathbf{B}_{1}, \ldots, \mathbf{B}_{\mathbf{n}} \Rightarrow \square \mathbf{L} . \square \mathbf{L} \supset \square \square \mathbf{L}-$ теорема. (Используем схему аксиом $\square \mathbf{A} \supset \square \square \mathbf{A}$.)

Тогда $\mathbf{B}_{\mathbf{1}}, \ldots, \mathbf{B}_{\mathbf{n}} \Rightarrow \square \square \mathbf{L}$.

Пусть $\mathbf{F}$ имеет значение $\mathbf{f}^{\mathbf{i}}$ в каждой альтернативной интерпретации, образованной на основе данной интерпретации. Тогда $\mathbf{L}$ имеет то же значение в каждой альтернативной интерпретации. В силу индукционного допущения $\mathbf{B}_{1}, \ldots, \mathbf{B}_{\mathbf{n}} \Rightarrow \neg \vee \mathbf{L}$. Тогда $\mathbf{B}_{1}, \ldots, \mathbf{B}_{\mathbf{n}} \Rightarrow \neg \diamond \square \mathbf{L}$. Используем схему аксиом $\diamond \square \mathbf{A} \supset \diamond \mathbf{A}$.

Пусть в некоторых альтернативных интерпретациях $\mathbf{F}$ имеет значение $\mathbf{f}^{\mathbf{c}}$, в некоторых - $\mathbf{f}^{\mathbf{i}}$. Тогда следует рассмотреть три возможности. Первая: $\mathbf{L}$ имеет значение $\mathbf{t}^{\mathbf{c}}$ в каждой альтернативной интерпретации. Вторая: $\mathbf{L}$ имеет значение $\mathbf{f}^{\mathbf{c}}$ в каждой альтернативной интерпретации. Третья: в некоторых альтернативных интерпретациях $\mathbf{L}$ имеет значение $\mathbf{t}^{\mathbf{c}}$, а в некоторых - $\mathbf{f}^{\mathbf{c}}$. Требуется доказать:

$\mathbf{B}_{1}, \ldots, \mathbf{B}_{\mathbf{n}} \Rightarrow(\neg \square \mathbf{L} \& \nabla \mathbf{L}) \vee \neg \diamond \square \mathbf{L}$, т. е. что $\mathbf{B}_{\mathbf{1}}, \ldots, \mathbf{B}_{\mathbf{n}} \Rightarrow \neg \square \mathbf{L} \vee \neg \nabla \mathbf{L}$.

При первой возможности сила индукционного допущения

$\mathbf{B}_{1}, \ldots, \mathbf{B}_{\mathbf{n}} \Rightarrow \mathbf{L} \& \diamond \neg \mathbf{L} . \diamond \neg \mathbf{L} \supset \neg \square \mathbf{L}-$ теорема. Доказано.

При второй возможности $\mathbf{B}_{1}, \ldots, \mathbf{B}_{\mathbf{n}} \Rightarrow \neg \mathbf{L} \& \diamond \mathbf{L}$. Доказано. (Используем аксиому $\square \mathbf{A} \supset \mathbf{A . )}$

При третьей возможности $\mathbf{B}_{1}, \ldots, \mathbf{B}_{\mathbf{n}} \Rightarrow(\mathbf{L} \& \diamond \neg \mathbf{L}) \vee(\neg \mathbf{L} \& \diamond \mathbf{L})$. Доказывается разбором случаев.

Случай 3. Пусть $\mathbf{s + 1 - м ~ в х о ж д е н и е м ~ л о г и ч е с к и х ~ т е р м и н о в ~ в ~ ф о р - ~}$ мулу $\mathbf{F}$ является вхождение знака возможности. Формула $\mathbf{F}$ есть $\diamond \mathbf{L}$.

Пусть $\mathbf{F}$ имеет значение $\mathbf{t}^{\mathbf{n}}$ в каждой альтернативной интерпретации, образованной на основе данной интерпретации переменных. Тогда $\mathbf{L}$ имеет значение $\mathbf{t}^{\mathbf{n}}$ в каждой альтернативной интерпретации. 
Требуется доказать: $\mathbf{B}_{\mathbf{1}}, \ldots, \mathbf{B}_{\mathbf{n}} \Rightarrow \square \backslash \mathbf{L}$. В силу индукционного допущения $\mathbf{B}_{\mathbf{1}}, \ldots, \mathbf{B}_{\mathbf{n}} \Rightarrow \square \mathbf{L} . \square \mathbf{A} \supset \square \vee \mathbf{A}$ - аксиома. Доказано.

Пусть $\mathbf{F}$ имеет значение $\mathbf{f}^{\mathbf{i}}$ в каждой альтернативной интерпретации, образованной на основе данной интерпретации переменных. Тогда $\mathbf{L}$ имеет значение $\mathbf{f}^{\mathbf{i}}$ в каждой альтернативной интерпретации. Требуется доказать: $\mathbf{B}_{\mathbf{1}}, \ldots, \mathbf{B}_{\mathbf{n}} \Rightarrow \neg \diamond \diamond \mathbf{L}$. В силу индукционного допущения $\mathbf{B}_{1}, \ldots, \mathbf{B}_{\mathbf{n}} \Rightarrow \neg \diamond \mathbf{L} . \diamond \diamond \mathbf{A} \supset \diamond \mathbf{A}-$ аксиома. Доказано.

Пусть в одних альтернативных интерпретациях, образованных на основе данной интерпретации переменных, $\mathbf{F}$ имеет значение $\mathbf{t}^{\mathbf{c}}$, в других - $\mathbf{t}^{\mathrm{n}}$. Тогда следует рассмотреть три возможности. Первая: $\mathbf{L}$ имеет значение $\mathbf{t}^{\mathrm{n}}$ в каждой альтернативной интерпретации. Вторая: $\mathbf{L}$ имеет значение $\mathbf{f}^{\mathbf{n}}$ в каждой альтернативной интерпретации. Третья: в одних альтернативных интерпретациях $\mathbf{L}$ имеет значение $\mathbf{t}^{\mathbf{c}}$, а в других - $\mathbf{f}^{\mathfrak{c}}$. Требуется доказать: $\mathbf{B}_{\mathbf{1}}, \ldots, \mathbf{B}_{\mathbf{n}} \Rightarrow(\diamond \mathbf{L} \& \diamond \neg \mathbf{L}) \vee \square \diamond \mathbf{L}$, т. е., что $\mathbf{B}_{\mathbf{1}}, \ldots, \mathbf{B}_{\mathbf{n}} \Rightarrow \oslash \mathbf{L}$. Несложное доказательство опускается.

Случай 4. s+1-м вхождением логических терминов в формулу $\mathbf{F}$ является вхождение знака импликации. Формула $\mathbf{F}$ есть $\mathbf{L} \supset \mathbf{M}$.

Пусть формула $\mathbf{F}$ имеет значение $\mathbf{t}^{\mathbf{n}}$ в каждой альтернативной интерпретации, образованной на основе данной интерпретации переменных. Это возможно, если в каждой альтернативной интерпретации $\mathbf{L}$ имеет значение $\mathbf{f}^{\prime}$ или в каждой альтернативной интерпретации $\mathbf{M}$ имеет значение $\mathbf{t}^{\mathrm{n}}$, или в некоторых альтернативных интерпретациях $\mathbf{L}$ имеет значение $\mathbf{f}^{\mathbf{i}}$, а в некоторых $\mathbf{M}-\mathbf{t}^{\mathbf{n}}$. Требуется доказать, что $\mathbf{B}_{1}, \ldots, \mathbf{B}_{\mathbf{n}} \Rightarrow \square(\mathbf{L} \supset \mathbf{M})$.

В первом случае используем схему аксиом $\neg \nabla \mathbf{A} \supset \square(\mathbf{A} \supset \mathbf{B})$.

Во втором - $\square \mathbf{A} \supset \square(\mathbf{A} \supset \mathbf{B})$.

В третьем случае, в силу индукционного допущения,

$\mathbf{B}_{1}, \ldots, \mathbf{B}_{\mathbf{n}} \Rightarrow \neg \backslash \mathbf{L} \vee \square \mathbf{M}$. Рассуждая с разбором случаев, получаем

$\mathbf{B}_{1}, \ldots, \mathbf{B}_{\mathbf{n}} \Rightarrow \square(\mathbf{L} \supset \mathbf{M})$.

Доказано.

Пусть формула $\mathbf{F}$ имеет значение $\mathbf{f}^{\mathbf{i}}$ в каждой альтернативной интерпретации, образованной на основе данной интерпретации переменных. Это возможно, если в каждой альтернативной интерпретации $\mathbf{L}$ имеет значение $\mathbf{t}^{\mathbf{n}}$, а $\mathbf{M}-\mathbf{f}^{\mathbf{i}}$. В силу индукционного допущения $\mathbf{B}_{1}, \ldots, \mathbf{B}_{\mathbf{n}} \Rightarrow \square \mathbf{L}$ и $\mathbf{B}_{1}, \ldots, \mathbf{B}_{\mathbf{n}} \Rightarrow \neg \mathbf{M}$. Используя аксиому $\diamond(\mathbf{A} \supset \mathbf{B}) \supset(\square \mathbf{A} \supset \diamond \mathbf{B})$, получаем $\mathbf{B}_{1}, \ldots, \mathbf{B}_{\mathbf{n}} \Rightarrow \neg \diamond(\mathbf{L} \supset \mathbf{M})$.

Пусть формула $\mathbf{F}$ имеет значение $\mathbf{t}^{\mathbf{c}}$ в каждой альтернативной интерпретации, образованной на основе данной интерпретации переменных. Это возможно в трех случаях. Первый — в каждой альтернативой интерпретации $\mathbf{L}$ имеет значение $\mathbf{t}^{\mathbf{n}}$, а $\mathbf{M}-\mathbf{t}^{\mathbf{c}}$. Второй - в каждой альтернативной интерпретации $\mathbf{L}$ имеет значение $\mathbf{f}^{\mathbf{c}}, \mathbf{a} \mathbf{M}-\mathbf{f}^{\mathbf{i}}$. Третий - в одних альтернативных интерпретациях $\mathbf{L}$ имеет значение $\mathbf{t}^{\mathbf{n}}$, а $\mathbf{M}-\mathbf{t}^{\mathbf{c}}$, в других $\mathbf{L}$ имеет значение $\mathbf{f}^{\mathbf{c}}$, а $\mathbf{M}-\mathbf{f}^{\mathbf{i}}$. Требуется доказать, что $\mathbf{B}_{\mathbf{1}}, \ldots, \mathbf{B}_{\mathbf{n}} \Rightarrow(\mathbf{L} \supset \mathbf{M}) \& \diamond \neg(\mathbf{L} \supset \mathbf{M})$. 
В силу индукционного допущения в первом случае

$\mathbf{B}_{1}, \ldots, \mathbf{B}_{\mathbf{n}} \Rightarrow \square \mathbf{L}$ и $\mathbf{B}_{1}, \ldots, \mathbf{B}_{\mathbf{n}} \Rightarrow \mathbf{M} \& \vee \mathbf{M} . \mathbf{M} \supset(\mathbf{L} \supset \mathbf{M})-$ теорема. Используя схему аксиом $\square(\mathbf{A} \supset \mathbf{B}) \supset(\square \mathbf{A} \supset \square \mathbf{B})$, получаем $(\square \mathbf{L} \& \diamond \neg \mathbf{M}) \supset \diamond \neg(\mathbf{L} \supset \mathbf{M})$.

Во втором случае в силу индукционного допущения верно $\mathbf{B}_{1}, \ldots$, $\mathbf{B}_{\mathbf{n}} \Rightarrow \neg \mathbf{L} \& \diamond \mathbf{L}$ и $\mathbf{B}_{1}, \ldots, \mathbf{B}_{\mathbf{n}} \Rightarrow \neg \diamond \mathbf{M} . \neg \mathbf{L} \supset(\mathbf{L} \supset \mathbf{M})-$ теорема. Используя схему аксиом $\square(\mathbf{A} \supset \mathbf{B}) \supset(\diamond \mathbf{A} \supset \diamond \mathbf{B})$, получаем

$(\diamond \mathbf{L} \& \diamond \neg \mathbf{M}) \supset \diamond \neg(\mathbf{L} \supset \mathbf{M})$.

Доказано.

При третьей возможности рассуждаем с разбором случаев.

Пусть $\mathbf{F}$ имеет значение $\mathbf{f}^{\mathbf{c}}$ в каждой альтернативной интерпретации, образованной на основе данной интерпретации переменных. Это возможно в трех основных случаях, а также в их сочетаниях:

1) в каждой альтернативной интерпретации $\mathbf{L}$ имеет значение $\mathbf{t}^{\mathbf{n}}$, a $\mathbf{M}-\mathbf{f}^{\mathbf{c}}$;

2) в каждой альтернативной интерпретации $\mathbf{L}$ имеет значение $\mathbf{t}^{\mathbf{c}}$, a $\mathbf{M}-\mathbf{f}^{\mathbf{c}}$;

3) в каждой альтернативной интерпретации $\mathbf{L}$ имеет значение $\mathbf{t}^{\mathbf{c}}$, a $\mathbf{M}-\mathbf{f}^{\mathbf{i}}$.

Требуется доказать, что

$\mathbf{B}_{1}, \ldots, \mathbf{B}_{\mathbf{n}} \Rightarrow \neg(\mathbf{L} \supset \mathbf{M}) \& \diamond(\mathbf{L} \supset \mathbf{M})$.

B первом случае в силу индукционного допущения имеет место: $\mathbf{B}_{1}, \ldots, \mathbf{B}_{\mathbf{n}} \Rightarrow \square \mathbf{L}$ и $\mathbf{B}_{1}, \ldots, \mathbf{B}_{\mathbf{n}} \Rightarrow \neg \mathbf{M} \& \diamond \mathbf{M}$. Далее $\square \mathbf{L} \Rightarrow \mathbf{L}$; $\mathbf{L} \& \neg \mathbf{M} \Rightarrow \neg(\mathbf{L} \supset \mathbf{M})$. Используя схему аксиом $\diamond \mathbf{B} \supset \diamond(\mathbf{A} \supset \mathbf{B})$, получаем требуемое доказательство.

Во втором случае в силу индукционного допущения будет верно: $\mathbf{B}_{1}, \ldots, \mathbf{B}_{\mathbf{n}} \Rightarrow \mathbf{L} \& \diamond \neg \mathbf{L}$ и $\mathbf{B}_{1}, \ldots, \mathbf{B}_{\mathbf{n}} \Rightarrow \neg \mathbf{M} \& \diamond \mathbf{M}$.

Доказано, как и в предшествующем случае.

В третьем случае в силу индукционного допущения имеет место: $\mathbf{B}_{1}, \ldots, \mathbf{B}_{\mathbf{n}} \Rightarrow \mathbf{L} \& \neg_{\mathbf{L}}$ и $\mathbf{B}_{1}, \ldots, \mathbf{B}_{\mathbf{n}} \Rightarrow \neg \backslash \mathbf{M}$. Доказано с использованием схемы аксиом $\diamond \neg \mathbf{A} \supset \diamond(\mathbf{A} \supset \mathbf{B})$.

Доказательство альтернативных случаев опускается.

Пусть формула $\mathbf{F}$ в одних альтернативных интерпретациях, образованных на основе данной интерпретации переменных, имеет значение $\mathbf{t}^{\mathbf{n}}$, а в других - $\mathbf{t}^{\mathbf{c}}$. Это возможно в трех основных случаях и их сочетаниях.

В первом случае как $\mathbf{L}$, так и $\mathbf{M}$ имеют значение $\mathbf{t}^{\mathbf{c}}$ в каждой альтернативной интерпретации. В силу индукционного допущения $\mathbf{B}_{1}, \ldots, \mathbf{B}_{\mathbf{n}} \Rightarrow \mathbf{L} \& \diamond \neg \mathbf{L}$ и $\mathbf{B}_{1}, \ldots, \mathbf{B}_{\mathbf{n}} \Rightarrow \mathbf{M} \& \oslash \neg \mathbf{M}$.

Требуется доказать:

$\mathbf{B}_{1}, \ldots, \mathbf{B}_{\mathbf{n}} \Rightarrow((\mathbf{L} \supset \mathbf{M}) \& \oslash \neg(\mathbf{L} \supset \mathbf{M})) \vee \square(\mathbf{L} \supset \mathbf{M})$, т. е. что $\mathbf{B}_{1}, \ldots, \mathbf{B}_{\mathbf{n}} \Rightarrow \mathbf{L} \supset \mathbf{M}$. 
Почти очевидное доказательство этого случая, а также других случаев и их сочетаний опускается.

Лемма доказана.

Доказательство метатеоремы 2. Поскольку формула $\mathbf{F}$ является общезначимой, то для любого набора гипотез $\mathbf{B}_{\mathbf{1}}, \ldots, \mathbf{B}_{\mathbf{n}}$ для нее верно $\mathbf{B}_{1}, \ldots, \mathbf{B}_{\mathbf{n}} \Rightarrow \square \mathbf{F}$, или же $\mathbf{B}_{1}, \ldots, \mathbf{B}_{\mathbf{n}} \Rightarrow \mathbf{F} \& \diamond \neg \mathbf{F}$. Отсюда $\mathbf{B}_{1}, \ldots, \mathbf{B}_{\mathbf{n}} \Rightarrow \mathbf{F}$. Остается показать, как удалить гипотезы:

1) $\mathbf{B}_{1}, \ldots, \mathbf{B}_{\mathbf{n}-\mathbf{1}}, \square \boldsymbol{\alpha}_{\mathbf{n}} \Rightarrow \mathbf{D}$;

2) $\mathbf{B}_{1}, \ldots, \mathbf{B}_{\mathbf{n}-\mathbf{1}}, \neg \nabla \boldsymbol{\alpha}_{\mathbf{n}} \Rightarrow \mathbf{D}$

3) $B_{1}, \ldots, B_{n-1}, \alpha_{n} \& \diamond \neg \alpha_{n} \Rightarrow D$;

4) $B_{1}, \ldots, B_{n-1}, \neg \alpha_{n} \& \backslash \alpha_{n} \Rightarrow D$.

Отсюда:

5) $\mathbf{B}_{1}, \ldots, \mathbf{B}_{\mathbf{n}-1}, \alpha_{\mathbf{n}}, \neg \neg \neg \alpha_{\mathbf{n}} \Rightarrow D$ (из 1$)$;

6) $\mathbf{B}_{1}, \ldots, \mathbf{B}_{\mathbf{n}-1}, \neg \alpha_{\mathrm{n}}, \neg \nabla \alpha_{\mathbf{n}} \Rightarrow \mathbf{D}$ (из 2);

7) $\mathbf{B}_{1}, \ldots, \mathbf{B}_{\mathbf{n}-1}, \alpha_{\mathbf{n}}, \nabla_{\neg} \alpha_{\mathbf{n}} \Rightarrow \mathbf{D}$ (из 3); Далее:

8) $\mathbf{B}_{1}, \ldots, \mathbf{B}_{\mathbf{n}-1}, \neg \alpha_{\mathbf{n}}, \diamond \boldsymbol{\alpha}_{\mathbf{n}} \Rightarrow \mathbf{D}$ (из 4).

9) $\mathbf{B}_{1}, \ldots, \mathbf{B}_{\mathbf{n}-1}, \boldsymbol{\alpha}_{\mathbf{n}} \Rightarrow \mathbf{D}$ (из 5, 7);

10) $\mathbf{B}_{1}, \ldots, \mathbf{B}_{\mathbf{n}-1}, \neg \boldsymbol{\alpha}_{\mathbf{n}}, \Rightarrow \mathbf{D}$ (из 6,8 );

11) $\mathbf{B}_{1}, \ldots, \mathbf{B}_{\mathbf{n}-\mathbf{1}} \Rightarrow \mathbf{D}($ из 9,10$)$ и т. д.

Метатеорема доказана.

Формализация основных квазматричных четырехзначных логик и доказательство соответствующих метатеорем проводятся следующим образом: проверяются на общезначимость приведенные в рассмотренной выше системе схемы аксиом. Те из них, которые не являются общезначимыми, отбрасываются. Начинается доказательство метатеорем о полноте. По мере потребности в новых схемах аксиом они включаются в аксиоматику, проверяясь предварительно на общезначимость. Затем, если возможно, минимизируется число схем аксиом, если удается показать, что некоторые из них являются производными.

Во всех основных квазиматричных четырехзначных логиках определения отрицания и импликации идентичные. Все соответствующие исчисления содержат следующие схемы аксиом:

$\square \mathbf{A} \supset \mathbf{A} ; \neg \square \neg \mathbf{A} \supset \diamond \mathbf{A} ; \diamond \mathbf{A} \supset \neg \square \neg \mathbf{A} ; \neg \nabla \mathbf{A} \supset \square(\mathbf{A} \supset \mathbf{B}) ; \square \mathbf{B} \supset \square(\mathbf{A} \supset \mathbf{B}) ;$ $\diamond \mathbf{B} \supset \diamond(\mathbf{A} \supset \mathbf{B}) ; \diamond \neg \mathbf{A} \supset \diamond(\mathbf{A} \supset \mathbf{B}) ; \diamond(\mathbf{A} \supset \mathbf{B}) \supset(\square \mathbf{A} \supset \diamond \mathbf{B})$.

В логике $\mathbf{S}_{\mathbf{K}}^{+}$дополнительными схемами являются: $\square \mathbf{A} \supset \square \mathbf{A}$; $\diamond \square \mathbf{A} \supset \diamond \mathbf{A} ; \square \mathbf{A} \supset \square \vee \mathbf{A} ; \diamond \diamond \mathbf{A} \supset \diamond \mathbf{A}, \square(\mathbf{A} \supset \mathbf{B}) \supset(\square \mathbf{A} \supset \square \mathbf{B}) ;$ $\square(\mathbf{A} \supset \mathbf{B}) \supset(\diamond \mathbf{A} \supset \diamond \mathbf{B}) . \mathrm{B}$ логике $\mathbf{S}_{\mathbf{a}}^{+}-\square(\mathbf{A} \supset \mathbf{B}) \supset(\square \mathbf{A} \supset \square \mathbf{B})$; $\square(\mathbf{A} \supset \mathbf{B}) \supset(\nabla \mathbf{A} \supset \diamond \mathbf{B})$.

Исследование выполнено при финансовой поддержке РГНФ в рамках научного проекта № 15-03-00372. 


\section{ЛИТЕРАТУРА}

[1] Ивлев Ю.В. Табличное построение пропозициональной модальной логики. Вестник Московского университета. Серия 7: Философия, 1973, № 6, c. 51-61.

[2] Ivlev Yu.V. Quasi-Functional Logic and Logic of Propositional Attitudes. Filosophie und Logik. Frege-Kolloquien Jena 1989/1991. Herausgegeben von Werner Stelzner. Walter de Gruyter. Berlin, New York, 1993, pp. 200-204.

[3] Ivlev Yu.V. Quasi-matrix logic as paraconsistent logic of dubitable information. Logic and Logical Philosophy, 2000, vol. 8, pp. 91-97.

[4] Ivlev Yu.V. Quasi-matrix logic. Journal of Multiple-Valued Logic and Soft Computing. Old City Publishing, Inc. (United States), 2005, vol. 11, no. 3-4, pp. 239-252.

[5] Ivlev Yu.V. Generalization of Kalmar's method for quasi-matrix logic. Логические исследования, 2013, т. 19, с. 281-307.

[6] Rescher N. Many-valued logic. New York, McGraw-Hill, 1969, 359 p.

[7] Ивлев Ю.В. Содержательная семантика модальной логики. Москва, Издательство Московского университета, 1985, 168 с.

[8] Ивлев Ю.В. Модальная логика. Москва, Издательство Московского университета, 1991, 224 с.

Статья поступила в редакцию 29.09.2016

Ссылку на эту статью просим оформлять следующим образом:

Ивлев Ю.В. Основные квазиматричные логики. Гуманитарный вестник, 2016, вып. 10. http://dx.doi.org/10.18698/2306-8477-2016-10-391

Ивлев Юрий Васильевич - д-р филос. наук, профессор кафедры логики философского факультета МГУ им. М.В. Ломоносова, заслуженный профессор МГУ, заслуженный работник Высшей школы РФ, лауреат Ломоносовской премии МГУ, академик PAEH. e-mail: ivlev.logic@yandex.ru 


\title{
Basic quasi-matrix logics
}

\author{
(C) Yu.V. Ivlev
}

Lomonosov Moscow State University, Moscow, 119991, Russia

Possibilities of applying matrix logics outside the scope of logics are known. They are relay-contact schemes and abstract automata. In case of applying matrix logics to the description of the abstract automata, dependencies of input automata signals on its output conditions and actions are expressed by means of functions. Quasi-matrix logics makes it possible to express these dependences using quasi-functions. Since a function is a particular case of a quasi-function, there appears a possibility of developing new kinds of automata applicable to a broader range of problems. However, since the decision problem of quasi-matrix logics is not still solved, quasi-matrix logics cannot be used in the considered area. The solution of this problem is given in the article.

Keywords: matrix logics, quasi-matrix logics, quasi-function, semantic completeness, decidability of calculus

\section{REFERENCES}

[1] Ivlev Yu.V. Vestnik Moskovskogo Universiteta. Seriya 7: Filosofiya - Bulletin of Lomonosov Moscow State University. Series 7: Philosophy, 1973, no. 6, pp. 51-61.

[2] Ivlev Yu.V. Filosophie und Logik. Frege-Kolloquien Jena 1989/1991. Herausgegeben von Werner Stelzner. Walter de Gruyter, Berlin. New York, 1993, pp. 200-204.

[3] Ivlev Yu.V. Logic and Logical Philosophy, 2000, vol. 8, pp. 91-97.

[4] Ivlev Yu.V. Journal of Multiple-Valued Logic and Soft Computing, 2005, vol. 11, no. 3-4, pp. 239-252.

[5] Ivlev Yu.V. Logical investigations, 2013, vol. 19, pp. 281-307.

[6] Rescher N. Many-valued logic. New York, McGraw-Hill, 1969, 359 p.

[7] Ivlev Yu.V. Soderzhatelnaya semantika modalnoy logiki [Contentive semantic of modal logics]. Moscow, Moscow Univ. Publ., 1985, 170 p.

[8] Ivlev Yu.V. Modalnaya logika [Modal logics]. Moscow, Moscow Univ. Publ., 1991, 224 p.

Ivlev Yu.V., Dr. Sci. (Philos.), Professor, Faculty of Philosophy, Department of Logics, Professor Emeritus of Lomonosov Moscow State University, Honorary Figure of Russian Higher Education, Lomonosov Moscow State University Award Winner, Member of the Russian Academy of Natural Sciences. e-mail: ivlev.logic@yandex.ru 